\title{
Anti-fouling ultrafiltration membrane prepared from polysulfone-graft-methyl acrylate copolymers by UV-induced grafting method
}

\author{
HUA Helin ${ }^{1,2}$, LI Na ${ }^{2}$, WU Linlin ${ }^{1}$, ZHONG Hui ${ }^{1}$, WU Guangxia ${ }^{1, *}$, \\ YUAN Zonghuan ${ }^{3}$, LIN Xiangwei ${ }^{3}$, TANG Lianyi ${ }^{3}$ \\ 1. Research Center for Eco-Environmental Science, Chinese Academy of Sciences, Beijing 100085, China. E-mail: huahelin76@yahoo.com.cn
2. Department of Environment and Chemistry Engineering, Nanchang Hangkong University, Nanchang 330034, China \\ 3. The Greatwall Pharmaceutical Factory of Beijing, Beijing 100071, China
}

Received 27 July 2007; revised 9 October 2007; accepted 14 November 2007

\begin{abstract}
Membrane fouling is one of the most important challenges faced in membrane ultrafiltration operations. The copolymers of polysulfone-graft-methyl acrylate were synthesized by homogeneous photo-initiated graft copolymerization. The variables affecting the degree of grafting, such as the time of UV (Ultraviolet-visible) irradiation and the concentrations of the methyl acrylate and photoinitiator, were investigated. The graft copolymer membranes were prepared by the phase inversion method. The chemical and morphological changes were characterized by attenuated total reflection-Fourier transform infrared spectroscopy (ATR/FT-IR), scanning electron microscopy, and water contact angles measurements. Results revealed that methyl acrylate groups were present on the membranes and the graft degree of methyl acrylate had remarkable effect on the performance of membranes. Pure water contact angle on the membrane surface decreases with the increase of methyl acrylate graft degree, which indicated that the hydrophilicity of graft copolymer membranes was improved. The permeation fluxes of pure water and bovine serum albumin solution were measured to evaluate the antifouling property of graft copolymer membranes, the results of which have shown an enhancement of antifouling property for graft copolymer membranes.
\end{abstract}

Key words: polysulfone; methyl acrylate; UV irradiation; graft polymerization

\section{Introduction}

Ultrafiltration membranes (UF) are used extensively in water/wastewater treatment, reverse osmosis pretreatment, and separations in the food, dairy, paper, textile, chemical, and biotechnology industries (Baker, 2004). However, the flux decline caused by concentration polarization and membrane fouling by proteins and other biomolecules adsorption in the feed stream is a repugnant challenge, which to a great extent prevents the wide-scale application in these industries (Marshall et al., 1993). Concentration polarization and membrane fouling not only decrease membrane permeability, but also shorten membrane life due to the aggressive chemicals necessary for cleaning. When cleaning becomes ineffective, the membranes must be replaced. Concentration polarization decreases the driving force of water flow across the membrane due to a local increase in foulant concentration. The effect is completely reversible, and can be reduced by modifying the flow over the membrane. Membrane fouling itself can occur in two ways: cake formation and absorption of foulants (Hilal et al., 2005). Cake fouling is generally reversible by water flushing or backwashing. However, fouling due

\footnotetext{
* Corresponding author. E-mail: wgxzhlj@yahoo.com.cn.
}

to the adsorption of foulants is essentially irreversible and can only be counteracted to a certain extent by aggressive chemical cleaning. Foulant adsorption can occur both on the membrane surface and in the pores. Since hydrophobic adsorption of foulants on membrane surface plays a key role in membrane fouling, hydrophilic modification of polymeric membrane surface can be one of the fouling mitigation methods (Pinnau et al., 2000). Many investigations have demonstrated that increasing membrane surface hydrophilicity could effectively inhibit membrane fouling in water/wastewater treatment. Therefore, various methods including blending, coating, adsorption, chemical-grafting, and radiation-induced grafting have been invented to modify membrane surface using hydrophilic modifiers (Roux et al., 2006; Meincken et al., 2005).

Polysulfone is very popular as an ultrafiltration membrane material. However, because of its hydrophobic nature, polysulfone membranes are susceptible to fouling by the adsorption of foulants (Song et al., 2004). Therefore, there are growing interests in developing hydrophilic polysulfone membrane surfaces. Several methods have been created to obtain hydrophilic polysulfone membrane surfaces, such as hydrophilic polymer coatings deposited onto the polysulfone membrane surface (Lee et al., 2000; 
Higuchi et al., 2003); chemical reaction of hydrophilic components onto the bulk polysulfone backbone (Summers et al., 2003; Lu et al., 2005; Park et al., 2006); hydrophilic layers graft-polymerized onto polysulfone membranes using radical reactions generated with low temperature plasma (Steen et al., 2001), gamma radiation (Mok et al., 1994) or UV irradiation (Kaeselev et al., 2001; Taniguchi et al., 2004).

A promising alternative approach to UF membrane surface modification that avoids extra processing steps is the addition of an amphiphilic component to the hydrophobic bulk material that can selectively segregate to the surface during membrane casting, producing a hydrophilic surface. For example, Chen et al. (1996) blended sulfonated or aminated polysulfone with polysulfone to obtain membranes with increased flux. Ishihara and coworkers (Ishihara et al., 1999) prepared polysulfone membranes incorporating phospholipidbased polymers and demonstrated reduced protein adsorption and low platelet adhesion relative to polysulfone. Kim and coworkers (Kim et al., 2005) employed polysulfone/poly (ethylene oxide) block copolymers as an additive in polysulfone UF membranes to improve hydrophilicity and protein fouling resistance.

In this study, the amphiphilic graft copolymers of polysulfone-graft-methyl acrylate were synthesized using homogeneous photo-initiated graft polymerization. The obtained graft copolymers with varying graft methyl acrylate content were cast for UF membranes using the phase inversion method. The graft methyl acrylate content, wettablity, pure water flux and fouling resistance of the membranes were investigated by attenuated total reflection-Fourier transform infrared spectroscopy (ATR/FT-IR), contact angle measurement and dead-end filtration. Scanning electron microscopy (SEM) was further performed to assess the influence of the degree of graft on membrane morphology. The membranes of the graft copolymers were then compared with the virgin polysulfone membrane for resistance to fouling by model protein (bovine serum albumin, BSA) solutions using dead-end filtration.

\section{Materials and methods}

\subsection{Materials}

Polysulfone (PSF, its specific viscosity is 0.73) was purchased from Dalian Polysulfone Plastic Co. Lt., China. Before use, it was dissolved in dichloromethane and precipitated into hexane. This purification procedure was repeated twice, and the final precipitate was dried under vacuum at $120^{\circ} \mathrm{C}$ for $12 \mathrm{~h}$. Dichloromethane $\left(\mathrm{CH}_{2} \mathrm{Cl}_{2}\right)$, methyl acrylate (MA), benzophenone (BP), ethyl ether and N-Methyl-2-pyrrolidinone (NMP) were purchased from Peking Chemistry and Reagent Co., China. Bovine serum albumin (BSA) was from Dongfang Instrument and Equipment Co., Chinese Academy of Sciences, China, and stored in safe. MA was distilled in vacuum to remove the polymerization inhibitor before use. Other reagents were used as received.

\subsection{Synthesis of PSF-graft-MA copolymers}

PSF-graft-MA (PSF- $g$-MA) copolymers were performed by irradiating homogeneous solutions of PSF, MA monomer and BP photoinitiator with UV light (the emission maximum at $365 \mathrm{~nm}$ and the intensity $20 \mathrm{~mW} / \mathrm{cm}^{2}$ ). Predetermined amounts of PSF, MA and BP were dissolved in tetrahydrofuran (THF) and the concentrated solutions so obtained were poured in a quartz vessel, and then the quartz vessel was placed in a photochemical reactor. To remove the dissolved oxygen from the solution, nitrogen gas was bubbled into the solution at a flow rate of $200 \mathrm{ml} / \mathrm{min}$ for $10 \mathrm{~min}$. The quartz vessel was irradiated with UV light at room temperature. After a predetermined time of graft copolymerization, the contents of the quartz vessel were precipitated in excess ethyl ether with stirring. The suspension obtained was filtered and then the solid product was vacuum dried to constant weight. The product was purified by dissolution in THF and precipitated in distilled water. The precipitates were washed with hot water to remove the homopolymer after filtration. This purification procedure was repeated twice. Finally, the product was vacuum dried at $80^{\circ} \mathrm{C}$ before analysis and use. The degree of grafting (DG) was calculated from the following equation:

$\operatorname{DG}(\%)=\left(\frac{W_{\mathrm{g}}-W_{0}}{W_{0}}\right) \times 100$

where, $W_{0}$ is the weight of initial PSF and $W_{\mathrm{g}}$ is the weight of graft copolymer after complete removal of unreacted monomer and homopolyer.

\subsection{Membrane casting}

The membranes were prepared from $15 \%(W / W)$ polymer (PSF or PSF- $g$-MA copolymer) solutions in NMP, then filtering and degassing under vacuum. Solutions were cast on a glass plate to form thin films. After exposure to air for $10 \mathrm{~s}$, the thin films were immersed in distilled water at $25^{\circ} \mathrm{C}$ for $2 \mathrm{~min}$. The obtained membrane was then placed in a distilled water bath overnight and stored in distilled water before use.

\subsection{Membrane characterization}

To investigate the chemical changes between the nascent membrane and graft copolymer membranes, attenuated total reflection-Fourier transform infrared (ATR/FT-IR) spectra with a Nicolet Avatar 360 FT-IR Spectrometer (Thermo Fisher Scientific Inc., USA) was used. Prior to the measurements, the samples were dried under a vacuum at $60^{\circ} \mathrm{C}$ for $24 \mathrm{~h}$. Data were recorded between $800-2,000$ $\mathrm{cm}^{-1}$ with 32 scans.

The morphology of the membranes, cross-section and surface were imaged with SEM (JSM-6301F, JEOL Ltd., Japan). Membranes were dried by Critical Point Dryer (EM Technologies Ltd., England) and then sputtered with gold. All SEM photographs were obtained under $5 \mathrm{keV}$ at $20^{\circ} \mathrm{C}$.

Contact angle measurements were performed on the cast membranes at $20^{\circ} \mathrm{C}$ with a CA-D contact angle meter (Kyowa Interface Science Co., Ltd., Japan) using de-ionized water as probe liquid. The membranes were 
fixed flat on a glass slide using double-sided tape and dried for $2 \mathrm{~h}$ in air and $2 \mathrm{~h}$ in a vacuum oven before the measurements. Five measurements were immediately taken after the droplet was placed on the membrane. Membrane wet ability was studied by placing $1 \mu \mathrm{l}$ of deionized water on the membrane surface, observing on the contact angle meter and measuring the time required for the water to be absorbed completely into the membrane. The values reported are averages of five measurements.

\subsection{Filtration experiments}

This process was conducted according to the report of Chen and Belfort (1999). The transmembrane pressure was kept constant at $0.1 \mathrm{MPa}$ and the filtration cell was stirred at $500 \mathrm{r} / \mathrm{min}$ using a stir plate to minimize concentration polarization. In a typical run, the solution reservoir was initially filled with de-ionized water, and the membrane was precompacted for $30 \mathrm{~min}$ during the filtration of de-ionized water. The permeation data were collected at fixed time intervals and weighed to determine transmembrane flux, until the flux remained constant for at least three successive readings. The average of five readings was recorded as $J_{0}$. Next, the reservoir was emptied and refilled with the model foulant solution. Protein solutions comprised $1000 \mathrm{mg} / \mathrm{L} \mathrm{BSA}$ in a phosphate-buffer solution (4.56 g NaH $2 \mathrm{PO}_{4}, 23.00 \mathrm{~g} \mathrm{Na}_{2} \mathrm{HPO}_{4}, 149.76 \mathrm{~g} \mathrm{NaCl}, 4.02$ $\mathrm{g} \mathrm{KCl}$, de-ionized water $1000 \mathrm{ml}, \mathrm{pH}$ 6.9). The flux of BSA filtration $\left(J_{1}\right)$ was recorded until the flux did not change any longer, which usually needed at least $40 \mathrm{~min}$. A sample of permeate was collected after $1 \mathrm{~h}$ of filtration. BSA retention values were obtained by measuring the foulant concentration in this sample using UV-visible spectroscopy with a LengGuang 752 ultraviolet-visible spectrophotometer (Shanghai Lengguang Technology Co., Ltd., China). The concentrations were quantified using UV absorbance at $280 \mathrm{~nm}$ for BSA. After that, the filtration cell was rinsed five to seven times with de-ionized water. Then the membrane was removed and inverted. De-ionized water was passed through the reversed membrane at 0.1 $\mathrm{MPa}$ pressure for $30 \mathrm{~min}$. Finally, the membrane was flipped back to its original orientation and the de-ionized water flux measure was designed as $J_{2}$.

\section{Result and discussion}

\subsection{Synthesis of PSF-g-MA copolymers}

UV-induced graft copolymerization is one of the most widely used techniques with vinyl monomers to natural and synthetic polymers. As we known, one of the most effective factors is the UV irradiation time. Therefore, the influence of UV irradiation time on the graft degree of MA was examined and the results are shown in Fig.1. It can be seen that the degree of graft (DG) of MA onto PSF increased progressively with UV irradiation time up to 10 min, and then decreased. This type of DG behaviour is generally observed in experiments carried out using photoinduced graft copolymerization. This may be explained by the fact that as the irradiation time increased, the number of grafting sites on the backbone increased. As a result,

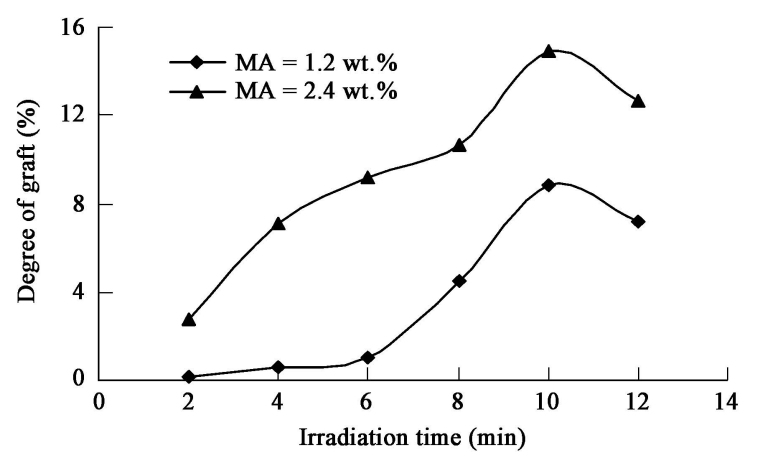

Fig. 1 Effect of the UV irradiation time on the degree of grafting (BP concentration $=0.25 \mathrm{wt} . \%$ ).

the extent of initiation and propagation of photografting copolymerization also increased with the irradiation time. However, beyond the maximum irradiation time, as the number of available sites for the photografting of monomer on the PSF backbone decreased, the reduction of DG was observed with the irradiation. Another possible explanation for the decrease in the DG at longer irradiation time is that the chain scissions of PSF are caused as a result of direct absorption of the incident radiation by the basic backbone structure of PSF.

The MA concentration is another key factor in relating to the graft degree of MA of graft copolymers and the typical results are listed in Table 1. The graft degree increased with increasing the MA concentration to a maximum value, and then leveled off. At a low monomer concentration, MA tends to graft onto the PSF backbone, causing the grafting percentage to increase at a given rate. With a further increase of the monomer concentration, the graft level dropped. This is because as the amount of monomer increases, there is an excess of monomer competing for the initiator, resulting in the homopolymerization and crosslink of the monomer as well as the shortage of available grafting sites.

\subsection{Membrane characterization}

The membrane surface was characterized by ATR/FT-IR spectroscopy and the typical spectra are depicted in Fig.2. The spectra of all membranes are similar at wavelengths below $1600 \mathrm{~cm}^{-1}$. The 1578 and $1486 \mathrm{~cm}^{-1}$ bands are due to the aromatic groups, and the $1323 \mathrm{~cm}^{-1} / 1289 \mathrm{~cm}^{-1}$ doublet and $1151 \mathrm{~cm}^{-1}$ band are assigned to the aromatic sulfone chromophore. Compared the graft copolymer membranes with the nascent one, a new peak appears at

Table 1 DG of PS-g-MA graft copolymer in various reaction conditions

\begin{tabular}{lllll}
\hline Polymer & \multicolumn{3}{c}{ Reaction conditions } & $\begin{array}{l}\text { DG } \\
\text { (wt.\%) }\end{array}$ \\
\cline { 2 - 4 } & $\begin{array}{l}\text { PSF:MA } \\
\text { (mass ratio) }\end{array}$ & $\begin{array}{l}\text { UV irradia- } \\
\text { tion time (min) }\end{array}$ & $\begin{array}{l}\text { BP in reac- } \\
\text { tion (wt.\%) }\end{array}$ & \\
\hline PSF- -MA1 & $1: 0.16$ & 6 & 0.12 & 2.95 \\
PSF- $g$-MA2 & $1: 0.32$ & 8 & 0.25 & 7.07 \\
PSF- $g$-MA3 & $1: 0.48$ & 8 & 0.25 & 10.62 \\
PSF- $g$-MA4 & $1: 0.64$ & 10 & 0.36 & 14.89 \\
PSF- $g$-MA5 & $1: 0.80$ & 10 & 0.36 & 12.35 \\
\hline
\end{tabular}

PS-g-MA: polysulfone-graft-methyl acrylate; DG: degree of graft; PSF: polysulfone; MA: methyl acrylate; BP: benzophenone. 


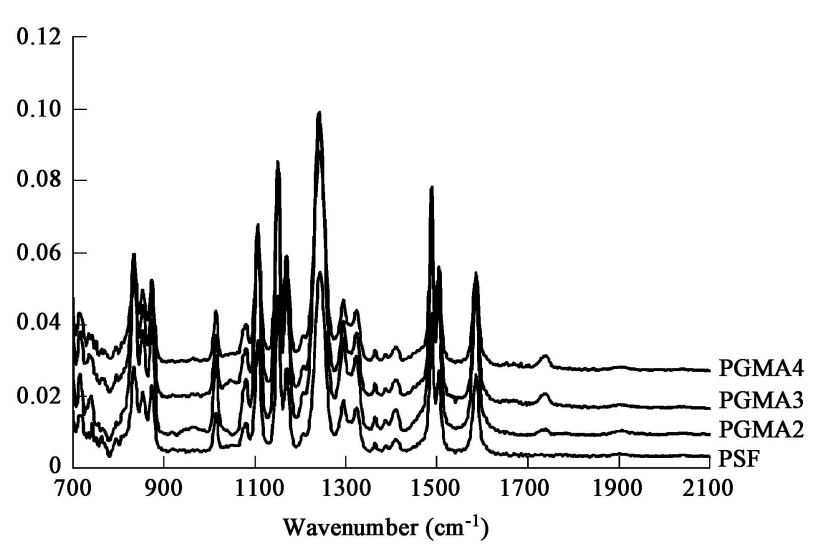

Fig. 2 ATR/FT-IR spectra of the membranes.

$1740 \mathrm{~cm}^{-1}$, which is corresponding to the typical absorption of carbonyl group in the MA. This new peak confirms the presence of MA on the surface of graft copolymer membranes. Moreover, with the increase of MA graft degree, the intensity of the peak becomes stronger.

Pure water fluxes of the nascent and graft copolymer membranes with the different graft degree are list in Table 2. The nascent PSF membrane exhibits low pure-water flux as compared with the graft copolymer membranes, indicating that the introduction of polar groups such as the carboxyl group does improve membrane permeability properties. This is because introducing MA onto the PSF backbone enhances the hydrophilicity of the membranes. The angle of water is generally used to evaluate the hydrophilicity of the membranes. As expected, all the graft copolymer membranes (Table 2) have lower contact angles than the nascent PSF membrane. With the increase of MA graft degree, the contact angle value decreases from $68^{\circ}$ to $51^{\circ}$. Referring to the FT-IR data (Fig.2), it can be inferred that the higher average degree of MA grafting on PSF result in membranes more hydrophilic. However, the smaller pure water flux values are found in the graft copolymer membranes of PGMA3 and PGMA4 as compared with PGMA2. In general, the variations in permeate flux data could be attributed to the degree of grafting, surface roughness, pore structure, pore sizes and pore size distribution. Thus, for PGMA2 membrane, the pure water flux is the highest, most probably as a result of larger pore sizes and pore density.

The SEM photographs reveal distinctly different morphological patterns for each of the membranes produced. The bottom view and the cross-sections of the membranes are shown in the SEM-micrographs of Fig.3. General structures of membranes are very similar, consisting of a thin dense layer at the top of the membrane, with an intermediate layer with a finger-like structure and porous architecture at the bottom. Morphologies of the ungrafted and grafted membranes are very similar, except that the amount of finger-like structure of graft copolymer membranes decreased and a larger finger-like type of structure is observed below the skin layer. In addition, the graft copolymer membranes with high graft degree show a relatively suppressed macropore structure and a much thicker sponge-like layer at the bottom of the cross section. The SEM analysis of the bottom view of the different membranes (Fig.3) shows that the photograph for
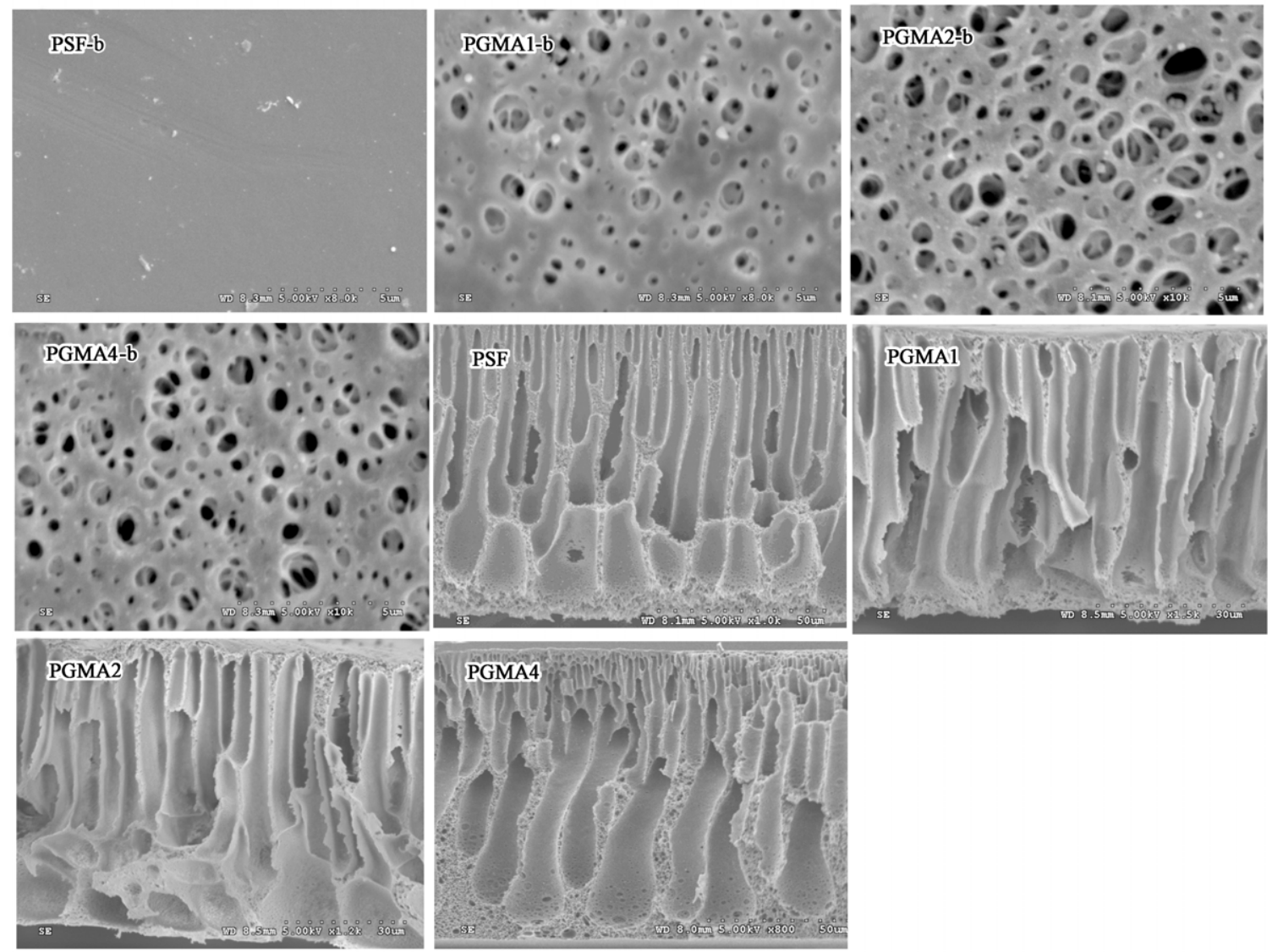

Fig. 3 SEM micrographs of the bottom view (b) and the cross-sections of the membranes (PSF, PGMA1, PGMA2, PGMA4). 
Table 2 Membrane compositions and performance characteristics

\begin{tabular}{llccc}
\hline Membrane & $\begin{array}{l}\text { Polymer } \\
\text { (in Table 1) }\end{array}$ & $\begin{array}{c}\text { Pure water permeability } \\
\left(\mathrm{L} /\left(\mathrm{m}^{2} \cdot \mathrm{h}\right)\right)\end{array}$ & $\begin{array}{c}\text { BSA retention } \\
\text { after } 1 \mathrm{~h}(\%)\end{array}$ & $\begin{array}{c}\text { Wetting } \\
\text { time }(\mathrm{min})\end{array}$ \\
\hline PSF & Neat PSF & 252 & 97.9 & 26 \\
PGMA1 & PSF-g-MA1 & 389 & 96.2 & 68 \\
PGMA2 & PSF-g-MA2 & 528 & 92.4 & 63 \\
PGMA3 & PSF-g-MA3 & 296 & 98.1 & 19 \\
PGMA4 & PSF-g-MA4 & 264 & 98.5 & 16 \\
\hline
\end{tabular}

the nascent PSF membrane is only a well defined smooth area. In the graft copolymer membranes, the Swiss-cheese morphology with smooth areas between pore openings is found. In contrast, a bottom view of the PGMA2 membrane shows more pronounced Swiss-cheese structures with different sizes and shapes as well as variable pore sizes between the polymer fragments.

\subsection{Permeation and antifouling property of the mem- branes}

The ATR/FT-IR, wetting, and contact angle results all revealed localization of PSF- $g$-MA to the membrane surfaces. To determine the effect on fouling resistance, the protein filtration was performed on the neat PSF, PGMA1, PGMA2, PGMA3 and PGMA4 with a feed solution containing $1000 \mathrm{mg} / \mathrm{L} \mathrm{BSA}$ in the phosphatebuffer solution. The results of these filtration runs as a function of the filtrate time are shown in Fig.4. As seen in the data, the flux decline behaviors during protein filtration were quite similar for all membranes. After $1 \mathrm{~h}$ filtration, the flux was reduced to $30 \%-50 \%$ of initial flux for all the membranes investigated, with higher flux declines

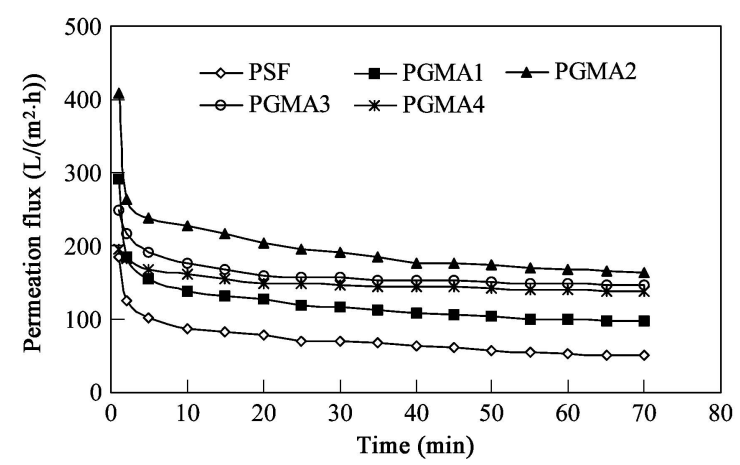

Fig. 4 Flux decline behavior of neat PSF and graft copolymer membranes during the BSA filtration. observed for membranes having higher initial flux. Furthermore, nascent PSF membrane showed a greater amount of flux decline compared to graft copolymer membranes. It is well known that membrane fouling can be influenced by hydrodynamic conditions, such as permeation drag and back transport, and chemical interaction between foulants and membranes. Since all the membranes were test at the same hydrodynamic condition, the different fouling behavior means that surface property of membrane was changed by MA component employment. Surface of graft copolymer membranes can be more hydrophilic than that of neat PSF membrane due to the hydrophilic MA component employed into the bulk PSF backbone. Therefore, hydrophobic adsorption between foulants and graft copolymer membranes was reduced, and deposited particles were readily removed by crossflow.

The permeation fluxes and flux changes during filtration for nascent PSF and graft copolymer membranes with different MA graft degree are shown in Fig.5. It can be seen in Fig.5a that almost all graft copolymer membranes experience both increase in the pure water and protein solution fluxes. When filtrated with a $1000 \mathrm{mg} / \mathrm{L} \mathrm{BSA} \mathrm{so-}$ lution, the membranes has an enhancing tendency toward antifouling property as the graft degree of MA increases, which is shown by the decrease of the total flux loss (1$\left.J_{0} / J_{1}\right)$, from $79 \%$ for the neat membrane to $47 \%$ for the PGMA4 membrane grafted with 14.89 wt.\% MA (Fig.5b).

The most notable differences between different polymer membranes were observed in the recovery step after a deionized water rinse. Cleaning the membrane was intended to remove BSA molecules adsorbed on the membrane surface, and the results of washing demonstrated the recovery ability of membrane fouled by protein. From the columns shown in Fig.5b, it can be seen that graft copolymer membranes are much more easily recovered with washing, and the overall water flux recovery $\left(J_{2}-J_{1}\right) /\left(J_{0}-J_{1}\right)$ is
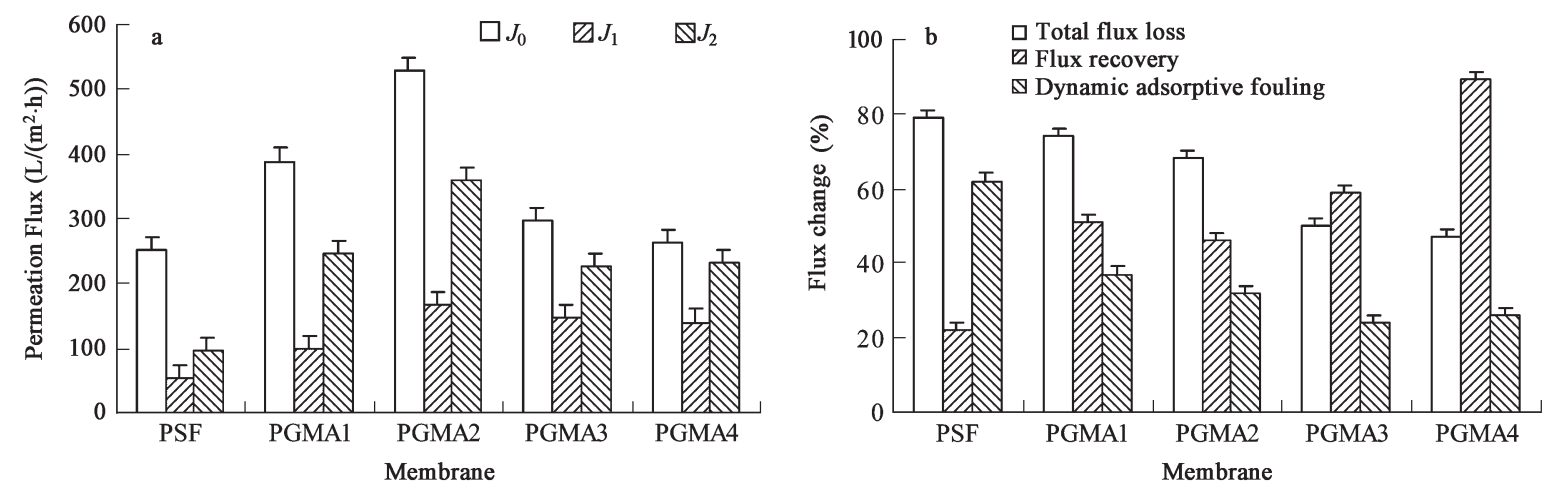

Fig. 5 Permeation fluxes (a) and flux changes (b) during filtration for neat PSF membrane and graft copolymer membranes with different MA graft degree. 
larger than that of nascent PSF membrane. For the PGMA4 membrane grafted with $14.89 \mathrm{wt} \%$, about $89 \%$ of the pure water flux loss was recovered after washing, indicating that a great part of BSA adsorptive fouling is reversible and the adhesion between the BSA and the graft copolymer membranes is small. From the data of dynamic adsorptive fouling $\left(1-J_{0} / J_{2}\right)$, the dynamic adsorptive fouling of nascent PSF is much higher than that of graft copolymer membranes, and the dynamic adsorption of BSA decreases with the increase of MA graft degree.

In summary, all graft copolymer membranes have higher pure water fluxes with enhanced flux recovery and reduced flux loss from BSA adsorptive fouling. Nevertheless, the PGMA2 membrane among the membranes studied has the highest $J_{0}, J_{1}$, and $J_{2}$, which indicates that at low graft degree the hydrophilicity has a major effect on the permeability, while this may mainly be overcompensated by the pore structure, sizes and distribution at high graft degree.

\section{Conclusions}

The PSF- $g$-MA copolymers were prepared by irradiating homogeneous solutions of PSF and MA with UV light. The DG of the MA groups onto the PSF polymers was affected by the UV irradiation time and the concentrations of both the MA monomer and photoinitiator. The membranes are cast by the phase inversion method. The ATR/FT-IR spectra and SEM photograph results demonstrate the chemical and morphological changes occurring on the membrane surface. Results from water contact angle measurements indicate that the hydrophilicity of graft copolymer membranes can be enhanced actually with the increase of graft degree. Pure water and BSA solution permeation results demonstrate the antifouling property of the membrane is improved as introducing the MA on the polysulfone boneback.

\section{Acknowledgements}

This work was supported by the National Natural Science Foundation of China (No. 20776148), the Science and Technology Program of Beijing (No. D0205004040421) and the Natural Science Foundation of Beijing (No. 2051002).

\section{References}

Baker R W, 2004. Membrane Technology and Application. Chichester: John Wily.

Chen H, Belfort G, 1999. Surface modification of poly(ether sulfone) ultrafiltration membranes by low-temperature plasmainduced graft polymerization. Journal of Applied Polymer Science, 72: 1999-2004.

Chen M H, Chiao T C, Tseng T W, 1996. Preparation of sulfonated polysulfone/polysulfone and aminated polysulfone/polysulfone blend membranes. Journal of Applied Polymer Science, 61: 1205-1209.

Higuchi A, Sugiyama K, Yoon B O, Sakurai M, Hara M, Sumita
M, Sugawara S, Shirai T, 2003. Serum protein adsorption and platelet adhesion on pluronic-adsorbed polysulfone membranes. Biomaterials, 24: 3235-3245.

Hilal N, Ogunbiyi O O, Miles N J, Nigmatullin R, 2005. Methods employed for control of fouling in MF and UF membranes: a comprehension review. Sep Sci Technol, 40: 1957-2005.

Ishihara K, Fukumoto K, Iwasaki Y, Nakabayashi N, 1999. Modification of polysulfone with phospholipid polymer for improvement of the blood compatibility. Part 2. Protein adsorption and platelet adhesion. Biomaterials, 20: 15531559.

Kaeselev B, Pieracci J, Belfort G, 2001. Photoinduced grafting of ultrafiltration membranes: comparison of poly(ether sulfone) and poly(sulfone). Journal of Membrane Science, 194: 245-261

Kim Y W, Ahn W S, Kim J J, Kim Y H, 2005. In situ fabrication of selftransformable and hydrophilic poly(ethylene glycol)modified polysulfone membranes. Biomaterials, 26: 28672875.

Lee J H, Ju Y M, Kim D M, 2000. Platelet adhesion onto segmented polyurethane film surfaces modified by addition and crosslinking of PEO containing block copolymers. Biomaterials, 21: 683-691.

Lu Z H, Liu G J, Duncanb S, 2005. Morphology and permeability of membranes of polysulfone-graft-poly(tert-butyl acrylate) and derivatives. Journal of Membrane Science, 250: 17-28.

Marshall A D, Muncro P A, Tragardh G, 1993. The effect of protein fouling in microfiltration and ultrafiltration on permeate flux, protein retention and selectivity: a literature review. Desalination, 91: 65-108.

Meincken M, Rtoux S, Jacobs E, 2005. Determination of the hydrophilic character of membranes by pulsed force mode atomic force microscopy. Appl Surf Sci, 252: 1772-1779.

Mok S, Worsfold D J, Founda A, Matsuura T, 1994. Surface modification of polyethersulfone hollow-fiber membranes by gamma-ray irradiation. Journal of Applied Polymer Science, 51: 193-199.

Park J Y, Acar M H, Akthakul A, Kuhlman W, Mayes A M, 2006. Polysulfone- graft-poly(ethylene glycol) graft copolymers for surface modification of polysulfone membranes. Biomaterials, 27: 856-865.

Pinnau I, Freeman B D, 2000. Membrane Formation and Modification, ACS Symposium Series 744, American Chemical Society, Washington DC.

Roux S P, Jacobs E, van Reenen A J, Morkel C, Meinckenl M, 2006. Hydrophilisation of polysulphone ultrafiltration membranes by incorporation of branched PEO-block-PSU copolymers. Journal of Membrane Science, 276: 8-15.

Song H, Wu G X, Liu K, 2004. Photochemical surface modification of poly(arylsulfone) ultrafiltration membrane and covalent immobilization of enzyme. Journal of Environmental Sciences, 16: 392-396.

Steen M L, Hymas L, Havey E D, Capps N E, Castner D G, 2001. Fisher E R. Low temperature plasma treatment of asymmetric polysulfone membranes for permanent hydrophilic surface modification. Journal of Membrane Science, 188: 97-114.

Summers G J, Ndawuni M P, Summers C A, 2003. Dipyridyl functionalized polysulfones for membrane production. Journal of Membrane Science, 226: 21-33.

Taniguchi M, Belfort G, 2004. Low protein fouling synthetic membranes by UV assisted surface grafting modification: varying monomer type. Journal of Membrane Science, 231: 147-157. 